\title{
Meal Data Domain
}

National Cancer Institute

\section{Source}

National Cancer Institute. Meal Data Domain. NCI Thesaurus. Code C49604.

A subject domain utilized for the submission of information encompassing and representing data, vocabulary or records related to meal data. 